\title{
Explorative Placebo-Controlled Double-Blind Intervention Study with Low Doses of Inhaled $\Delta$ 9-Tetrahydrocannabinol and Cannabidiol Reveals No Effect on Sweet Taste Intensity Perception and Liking in Humans
}

\author{
Suzanne E.M. de Bruijn, Cees de Graaf, Renger F. Witkamp, and Gerry Jager*
}

\begin{abstract}
Introduction: The endocannabinoid system (ECS) plays an important role in food reward. For example, in humans, liking of palatable foods is assumed to be modulated by endocannabinoid activity. Studies in rodents suggest that the ECS also plays a role in sweet taste intensity perception, but it is unknown to what extent this can be extrapolated to humans. Therefore, this study aimed at elucidating whether $\Delta$ 9-tetrahydrocannabinol (THC) or cannabidiol (CBD) affects sweet taste intensity perception and liking in humans, potentially resulting in alterations in food preferences.

Materials and Methods: In a randomized placebo-controlled, double-blind crossover study, 10 healthy males participated in three test sessions that were 2 weeks apart. During the test sessions, participants received THC-rich, CBDrich, or placebo Cannabis by inhalation divided over two doses ( $4+1 \mathrm{mg} \mathrm{THC} ; 25+10 \mathrm{mg}$ CBD). Participants tasted seven chocolate milk-like drinks that differed in sugar concentration and they rated sweet taste intensity and liking of the drinks. They were then asked to rank the seven drinks according to how much they liked the drinks and were offered ad libitum access to their favorite drink. In addition, they completed a computerized food preference task and completed an appetite questionnaire at the start, midway, and end of the test sessions.

Results: Inhalation of the Cannabis preparations did not affect sweet taste intensity perception and liking, ranking order, or ad libitum consumption of the favorite drink. In addition, food preferences were not influenced by the interventions. Reported fullness was lower, whereas desire to eat was higher throughout the THC compared to the CBD condition.

Conclusions: These results suggest that administration of Cannabis preparations at the low doses tested does not affect sweet taste intensity perception and liking, nor does it influence food preferences in humans.
\end{abstract}

Keywords: cannabidiol; food preferences; phytocannabinoids; sweet taste; tetrahydrocannabinol

\section{Introduction}

There is growing evidence that the endocannabinoid system (ECS) plays an important role in the regulation of hedonic hunger, that is, consuming foods for the experience of reward and not for the compensation of any energy deficit. ${ }^{1}$ More specifically, the ECS seems to modulate palatability-dependent appetite, ${ }^{2-5}$ although the underlying mechanisms are far from clear.

It is important to note that palatability, or pleasantness, is not a fixed function of the sensory properties

Division of Human Nutrition, Wageningen University, Wageningen, The Netherlands.

Parts of this study have previously been submitted as an abstract to the International Cannabinoid Research Society 2015 and to the Society for the Study of Ingestive Behavior 2015.

*Address correspondence to: Dr. Gerry Jager, Division of Human Nutrition, Wageningen University, P.O. Box 17, Wageningen 6700 AA, The Netherlands, E-mail: gerry.jager@wur.nl

(C) Suzanne E.M. de Bruijn et al. 2017; Published by Mary Ann Liebert, Inc. This is an Open Access article distributed under the terms of the Creative Commons Attribution License, which permits unrestricted use, distribution, and reproduction in any medium, provided the original work is properly cited. Mary Ann Liebert, Inc. offers reprint services for those who want to order professionally produced copies of articles published under the Creative Commons Attribution (CC BY) license. To obtain a price quote, email Reprints@liebertpub.com. Please include the article's title or DOI, quantity, and delivery destination in your email. 
(e.g., taste, smell, texture) of a food, but also depends on the hedonic evaluation of more qualitative (liking) and contextual (eating occasion) characteristics. ${ }^{6,7}$

Considering taste, there seem to be two ways in which taste intensity perception and liking can be modulated. One is through alterations in quantitative taste intensity perception, reflected by a shift in the psychophysical (concentration intensity) function. In case of, for example, sweet taste, the same sugar concentration is perceived as more or less intensely sweet. The other way is related to changes in hedonic evaluation, reflected by a shift in the psychohedonic (intensity pleasantness) function, where for example, the perceived sweetness intensity of a sugar concentration is unchanged, but the perceived sweetness is liked more or less. ${ }^{8}$

In rodents, endocannabinoid activity modulates sweet taste palatability. Endocannabinoid stimulation enhances liking of sweet taste $\mathrm{e}^{9,10}$ and motivational behavior aimed at obtaining a sucrose solution, ${ }^{11}$ whereas endocannabinoid inhibition reduces liking and motivational behavior. ${ }^{9,11}$ Some studies suggest that administration of cannabidiol (CBD) decreases food intake ${ }^{12,13}$ possibly through indirect antagonism of the ECS, ${ }^{14,15}$ whereas other studies do not find an effect of CBD on food intake. ${ }^{16,17}$ Taken together, it is clear that $\mathrm{CBD}$ is far from being a classical ligand for the $\mathrm{CB}_{1}$ or $\mathrm{CB}_{2}$ receptor. The molecule displays a wide spectrum of activities on various molecular targets and produces multiple pharmacological effects. ${ }^{15,18}$

Administration of endocannabinoids was found to specifically increase responses to sweet taste in the Chorda tympani, the nerve that innervates the tongue, in wild-type mice, but not in $\mathrm{CB}_{1}$ knockout mice. ${ }^{19}$ Administration of a $\mathrm{CB}_{1}$ antagonist decreased responses to sweet taste in the Chorda tympani. ${ }^{20}$ Together, these results suggest that modulating the ECS selectively changes the psychophysical function for sweet taste in mice.

In humans, anecdotal evidence suggests that the use of Cannabis preparations increases food palatability and intake, ${ }^{21-25}$ a phenomenon colloquially known as "the munchies." Furthermore, the $\mathrm{CB}_{1}$ inverse agonist rimonabant decreases food intake. ${ }^{26,27}$ Thus far, only one study examined the effects of oral administration of $\Delta 9$-tetrahydrocannabinol (THC) on taste intensity and liking in humans. In a double-blind placebo-controlled study, acute Cannabis administration was found not to affect taste intensity perception and liking for sweet, sour, salty, and bitter food stimuli at baseline, and 2, 4, and $6 \mathrm{~h}$ postdosing. However,
THC bioavailability varied widely and THC could not be detected in plasma of all participants. ${ }^{28}$ Based on the limited number of studies performed in humans, the aim of this study was to further investigate whether modulating the ECS by administering phytocannabinoid preparations affects sweet taste intensity perception and liking in humans, and whether this results in altered food preferences.

\section{Methods}

\section{Participants}

Ten healthy, normal weight (body mass index $=21.7 \pm$ $1.2 \mathrm{~kg} / \mathrm{m}^{2}$ ) males aged $23.4 \pm 1$ years participated in this study. Participants were incidental Cannabis users to ensure familiarity with the psychotropic effects of Cannabis use. Frequency of use ranged from four times a year to once a week. To limit the risk of adverse events, males were excluded from participation when they had ever experienced negative effects from using Cannabis (e.g., bad trip, Cannabis-induced psychosis). Other exclusion criteria were restraint eating (Dutch eating behavior questionnaire: score >2.9), ${ }^{29}$ lack of appetite, difficulties swallowing or eating, having taste or smell disorders (self-report), following an energy-restricted diet 2 months before the study, weight change $>5 \mathrm{~kg}$ during the 2 months before the study, and being allergic or intolerant to the products under study. Participants were instructed to abstain from Cannabis from 2 weeks before the first test session until study completion. Compliance was tested by means of a urine sample (SureScreen Diagnostics Ltd.) at the beginning of each test session. Participants were also asked not to use alcohol in the 2 days before a test session and not to eat or drink anything except water or weak tea during the $2 \mathrm{~h}$ before a test session. Participants gave written informed consent before the start of the study and they received financial compensation at the end of the study. The study was approved by the Medical Ethics Committee of Wageningen University and was conducted in accordance with the Helsinki declaration of 1975 , as revised in 2008. The study was registered on clinicaltrials.gov as NCT02112292.

\section{Design and procedures}

The study had a randomized, placebo-controlled, doubleblind crossover design. All participants completed one practice session (familiarization with the test procedures) and three test sessions after administration of Cannabis rich in THC, CBD, or placebo (for details see section "Study drugs and administration"). Test sessions were 2 weeks apart to allow for complete clearance of drugs. 
Each test session consisted of repeated measurements of appetite and subjective effects using Visual Analogue Scales (VAS), venous blood sampling to determine plasma concentrations of THC and $\mathrm{CBD}$, a taste test with chocolate milk-like drinks that differed in sugar concentration (for details see section "Sweet taste intensity perception and liking"), a control task, a ranking test with the drinks, ad libitum intake of the preferred chocolate milk-like drink, and the Macronutrient and Taste Preference Ranking Task (MTPRT). ${ }^{30}$ See Figure 1 for a schematic overview of the test procedure.

\section{Study drugs and administration}

The active preparations consisted of the dried, milled, and homogenized flowers of two varieties of the plant Cannabis sativa and were obtained from the Office for Medicinal Cannabis (OMC; The Netherlands), variety Bedrocan ${ }^{\circledR}(19 \% \mathrm{THC},<1 \% \mathrm{CBD})$ and variety Bedrolite $^{\circledR}(<1 \%$ THC, 9\% CBD). The placebo (variety "Bedrocan"; $<0.5 \%$ THC) had a moisture content and terpenoid profile (providing the typical smell and taste of Cannabis) identical to the active drug, and was obtained from Bedrocan BV (official supplier to the OMC). ${ }^{31}$ From here onward, we will refer to the three treatments as the THC (Bedrocan), CBD (Bedrolite), and placebo condition. In the THC condition, participants received an initial dose of $4 \mathrm{mg}$ of THC, followed by $1 \mathrm{mg}$ of THC as a top-up dose after 35 min to maintain stable levels of central nervous system effects. ${ }^{32}$ In the CBD condition, the initial and topup dose of CBD were 25 and $10 \mathrm{mg}$, respectively.

For each individual dose, exact amounts of Bedrocan or Bedrolite and placebo were mixed so that each dose was equal to $250 \mathrm{mg}$ of plant preparation total weight.
According to Good Manufacturing Practice guidelines and to ensure double-blindness, study medication was prepared, packed, and coded by ACE Pharmaceuticals BV (Zeewolde, The Netherlands), a company licenced to prepare trial medication. Study medication was stored at a temperature of $-18^{\circ} \mathrm{C}$ and taken out of the freezer $30 \mathrm{~min}$ before the start of a test session. Administration was performed with a MINIVAP vaporizer (Hermes Medical Engineering, S.L.) that was set at a temperature of $230^{\circ} \mathrm{C}$. For each administration, participants were instructed to inhale 12 times with a duration of $5 \mathrm{sec}$ per inhalation and a pause of $25 \mathrm{sec}$ between adjacent inhalations.

\section{Plasma levels and subjective effects}

Venous blood samples were collected in $\mathrm{NaF} / \mathrm{KOx}$ (anticoagulant) tubes at baseline ( $T=0), T=30$, and $T=75 \mathrm{~min}$, and stored at $4^{\circ} \mathrm{C}$ until the end of the test session. Then, tubes were centrifuged at $1300 \mathrm{~g}$ for $10 \mathrm{~min}$ at $4^{\circ} \mathrm{C}$ and plasma samples were collected and stored at $-80^{\circ} \mathrm{C}$ until analyses. Plasma was analyzed for THC and CBD concentrations $(\mathrm{ng} / \mathrm{mL})$ using liquid chromatography-mass spectrometry/mass spectrometry by Analytical Biochemical Laboratory BV (Assen, The Netherlands). Due to budget restraints, the samples of the placebo condition were only analyzed for half of the sample $(n=5)$.

Subjective effects of the treatments were assessed with a VAS on which participants rated "feeling high" throughout the test sessions.

\section{Sweet taste intensity perception and liking}

A series of chocolate milk-like drinks that differed in sugar concentration was used to assess sweet taste intensity perception and liking. Samples were prepared freshly on the

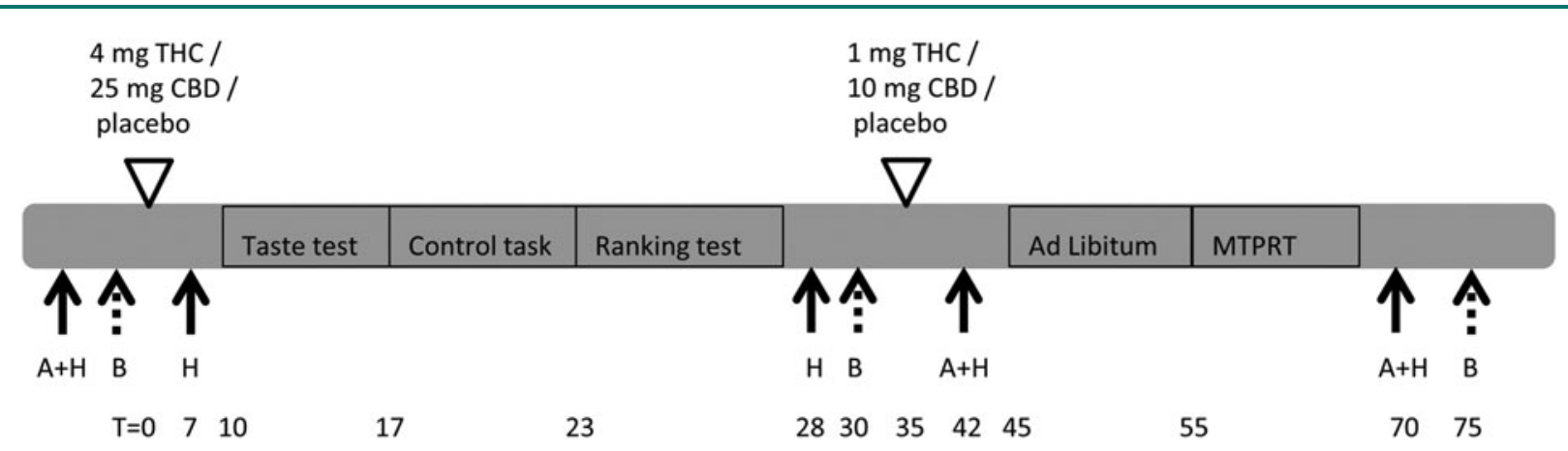

FIG. 1. Schematic overview of a test session. $A$, appetite questionnaire; $H$, feeling high (Visual Analogue Scale); B, blood sample; MTPRT, Macronutrient and Taste Preference Ranking Task; T, time; THC, $\Delta 9$ tetrahydrocannabinol; CBD, cannabidiol. 
Table 1. Composition of the Different Chocolate Milk-Like Drinks Per $100 \mathrm{~mL}$

\begin{tabular}{lccc}
\hline Added sugar $(\mathbf{g} / \mathbf{1 0 0} \mathbf{~ m L})$ & Total weight $(\mathbf{g})$ & Basic $(\mathbf{g})$ & Sugar $(\mathbf{g})$ \\
\hline 2 & 85 & 85 & 0 \\
4.4 & 86 & 83.6 & 2.4 \\
6.7 & 87 & 82.3 & 4.7 \\
10 & 88.6 & 80.6 & 8 \\
15 & 91.5 & 78.5 & 13 \\
22.5 & 95.7 & 75.2 & 20.5 \\
33.8 & 102.2 & 70.4 & 31.8 \\
\hline
\end{tabular}

The basic drink contained $2 \mathrm{~g} / 100 \mathrm{~mL}$ added vanilla sugar.

morning of each test session. First, a basic drink was prepared, containing vanilla sugar (Ruf; $2 \%)$, Nutilis powder (Nutricia; 3\%), cream (De Zaanse Hoeve; 18\%), skimmed milk (De Zaanse Hoeve; 74.5\%), and cocoa powder (Blooker; 2.5\%). Then, different amounts of basic chocolate milk-like drink and sucrose (Van Gilse) were mixed to get seven drinks with $2,4.4,6.7,10,15,22.5$, and $33.8 \mathrm{~g}$ of added sugar per $100 \mathrm{~mL}$, respectively (Table 1 ). These sugar concentrations were chosen to cover a full psychophysical sweetness intensity curve, ranging from not that sweet to very sweet. The drinks were coded with random three-digit numbers and presented to the participants in $20 \mathrm{~mL}$ samples, in a random order that differed between participants, but was kept constant per participant across test sessions. Participants were instructed to take a sip and then first rate sweet taste liking, followed by sweet taste intensity on a 200 -point labeled affective magnitude scale ${ }^{33}$ and a 100-point labeled magnitude scale, ${ }^{34}$ respectively. In between samples, participants were instructed to rinse their mouth with water and wait $30 \mathrm{sec}$ before tasting the next sample.

\section{Control task}

To check whether the Cannabis treatments did not affect task performance or perception in general, a control task was added to the procedures. Participants were asked to rate the grayness of seven shades of gray on a 100-point VAS that was anchored by "white" and "black." 8 Reflection percentages of the different shades were $96 \%, 84 \%$, $64 \%, 44 \%, 20 \%, 8 \%$, and $4 \%$, respectively, with shades ranging from almost white to almost black. Analyses showed that ratings did not differ between conditions and participants were able to differentiate between the different shades of gray (results not reported).

\section{Appetite, ranking task, and ad libitum intake}

Throughout the test sessions, participants thrice (see Fig. 1) rated levels of hunger, fullness, desire to eat, and prospective consumption on a 100-point VAS anchored by "not at all" and "extremely", except for prospective consumption, which was anchored by "nothing at all" and "a very large amount." 35

After the control task, participants were presented with $80 \mathrm{~mL}$ of the seven chocolate milk-like drinks and were asked to rank them according to how much they liked the drinks. During the ad libitum intake test, participants were presented with $1 \mathrm{~L}$ of their favorite drink, which they could consume ad libitum for $10 \mathrm{~min}$.

\section{Food preferences}

Food preferences were assessed using the MTPRT. In this task, participants were presented with four pictures of different food products at a time and asked to rank the products according to what they most desire to eat at that moment. Food products included in this task were high in carbohydrate, high in fat, high in protein, or low in energy, and had a sweet or savory taste. Examples of foods included in the food preference test are pancakes (highcarbohydrate sweet), salty sticks (high-carbohydrate savory), chocolate bar (high-fat sweet), cheese cubes (high-fat savory), shrimps (high-protein), strawberries (low-energy sweet), and cucumber (low-energy savory). In short, the task consists of a macronutrient section, in which participants are presented with one product from each of the four macronutrient categories, and a taste section, in which participants are presented with two sweet and two savory products. Based on the rankings, preference scores for different macronutrient and taste categories, that is, high-carbohydrate, high-fat, high-protein, and low-energy, and sweet and savory are calculated as described elsewhere. ${ }^{30}$ Preference scores for the macronutrient categories can range from 1 to 4 , with a higher score indicating a higher preference for a category. A score of 1 indicates all products from that category were ranked last and a score of 4 indicates all products from that category were ranked first. As each ranking in the taste section comprises two sweet and two savory products, preference scores for sweet and savory can range from 1.5 to 3.5. Because preference scores for sweet and savory are each other's opposite, we only report preference scores for sweet in this article.

\section{Data analyses}

Data are presented as mean with standard error of the mean. Data were analyzed using IBM SPSS 22 (IBM Corporation). Results were considered statistically significant at $p<0.05$. When statistically significant main effects were found, post-hoc tests were performed using Bonferroni correction. 
Table 2. 4 9-Tetrahydrocannabinol and Cannabidiol Plasma Levels for All Three Conditions

\begin{tabular}{lccc}
\hline Condition & Compound & $\boldsymbol{T}=\mathbf{3 0}$ & $\boldsymbol{T}=\mathbf{7 5}$ \\
\hline THC & THC & $1.6 \pm 0.5$ & $1.3 \pm 0.4$ \\
& CBD & $0.34 \pm 0.1$ & $0.26 \pm 0.1$ \\
CBD & THC & 0.16 & $0.21 \pm 0.03$ \\
& CBD & $2.8 \pm 0.8$ & $3.0 \pm 0.7$ \\
Placebo & THC & $0.22 \pm 0.05$ & $0.21 \pm 0.04$ \\
& CBD & ND & ND \\
\hline
\end{tabular}

Plasma levels in $\mathrm{ng} / \mathrm{mL}$. Values are mean \pm SEM. $n=10$ for THC in THC condition and $\mathrm{CBD}$ in $\mathrm{CBD}$ condition. $\mathrm{CBD}$ was detected in four participants $(T=30$ and $T=75)$ in the THC condition, THC was detected in one $(T=30)$ and three $(T=75)$ participants in the CBD condition. Placebo condition was tested for five participants. THC was detected in four $(T=30)$ and five $(T=75)$ participants.

THC, $\triangle 9$-tetrahydrocannabinol; CBD, cannabidiol; ND, not detected; SEM, standard error of the mean.

Liking ratings of the chocolate milk-like drinks were transferred to 100 points by dividing the scores by two. Using repeated measures analysis of variance (ANOVA), rated sweetness intensity and liking of the drinks were compared, with condition (THC, CBD, and placebo) and sugar concentration as factors. For the ranking task, the mean rank was calculated for each chocolate milk-like drink in each condition. Friedman ANOVA was used to compare mean ranks between conditions for each drink separately and ranks of the drinks within conditions. For the ad libitum task, the consumed amount and energy content of the consumed drink were used to calculate the consumed energy. Repeated measures ANOVA was used to test if the consumed energy differed between conditions.

Preference scores from the MTPRT were compared between conditions using repeated measures ANOVA. To compare preference scores within each condition, Friedman ANOVA was used for the macronutrient categories and Wilcoxon signed rank tests were used to compare the sweet and savory preference scores.

Appetite ratings were compared using repeated measures ANOVA with condition (THC, CBD, and placebo) and time as factors. Because of one missing data point for one participant, mixed models ANOVA was used to analyze "feeling high" with condition, time, and condition $\times$ time as fixed factors and subject as a random factor.

\section{Results}

Plasma levels and subjective effects

Table 2 shows the mean TCH and CBD plasma levels for all three conditions. In some participants, low levels of CBD were detected in the THC condition and vice versa. Low levels of THC were detected in plasma samples after administration of placebo, but these were 8 - versus 6.5 -fold lower compared to $T=30$ and $T=75 \mathrm{~min}$ in the THC condition.

As shown in Figure 2, reported levels of "feeling high" differed between conditions, $F(2,13)=4.60, p<0.05$, and over time, $F(4,22)=7.35, p<0.05$. Post-hoc analyses showed that participants felt significantly more "high" in the THC compared to the placebo condition.

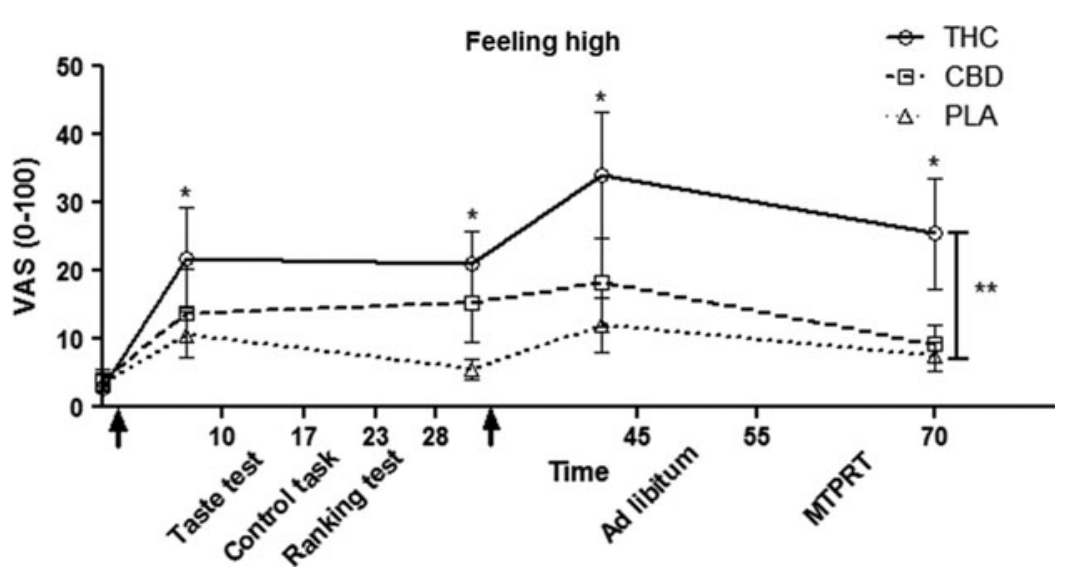

FIG. 2. Reported "feeling high" (mean \pm SEM). Arrows indicate treatment administration. For the first arrow: $4 \mathrm{mg} \mathrm{THC}, 25 \mathrm{mg}$ CBD or placebo. For the second arrow: $1 \mathrm{mg} \mathrm{THC}, 10 \mathrm{mg}$ CBD or placebo. *Indicates significant difference from $T=0(p<0.05)$. ${ }^{* *}$ Indicates difference between THC and placebo conditions $(p<0.05)$. PLA, placebo; SEM, standard error of the mean. 
Sweet taste intensity perception and liking

Drinks that contained more sugar were perceived as more sweet, $F(6,54)=57.87, p<0.05$, but the perceived sweet taste intensity of the seven chocolate milk-like drinks did not differ between the three conditions, $F(2,18)=$ $0.66, p=0.53$ (Fig. 3A). Liking of different drinks differed with sugar concentration, $F(6,54)=2.47, p<0.05$, where the drinks that contained the least and the most sugar were liked least. Similar to the intensity findings, the liking of the seven drinks did not differ between conditions, $F(2,18)=0.94, p=0.41$ (Fig. 3B).

\section{Ranking task}

Ranks of the different drinks did not differ between conditions, all $p>0.05$. In addition, the ranking task
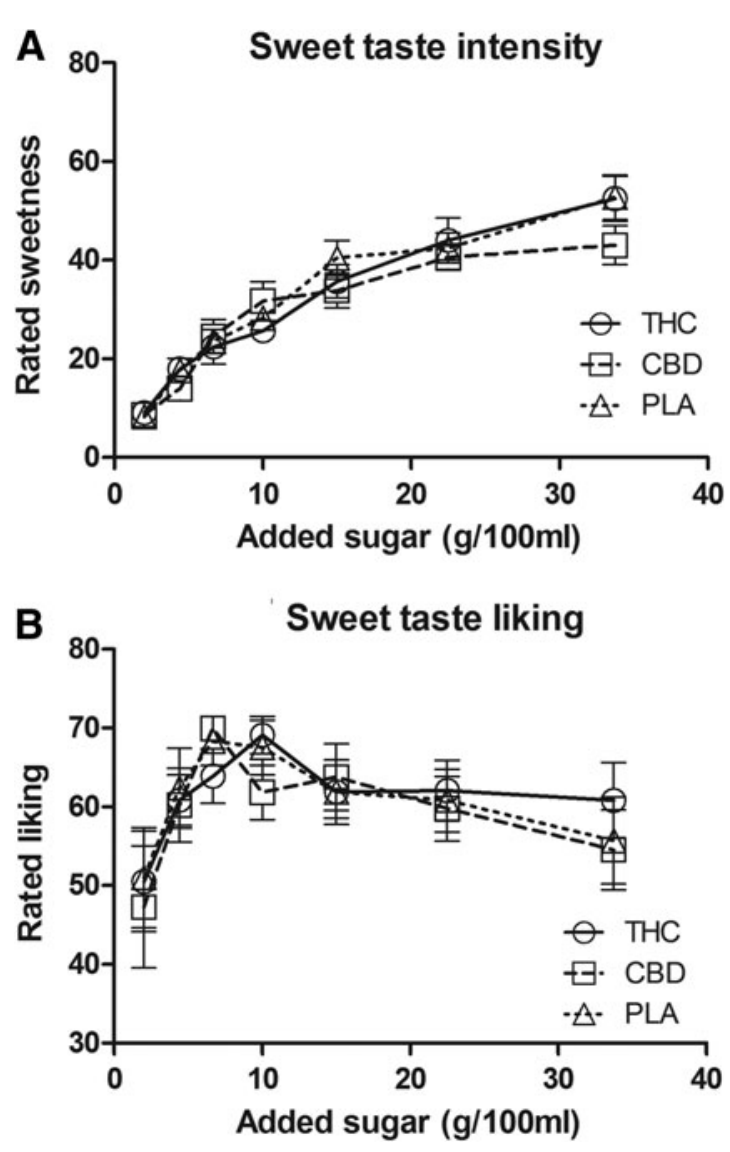

FIG. 3. Ratings (mean \pm SEM) for (A) sweet taste intensity, and (B) sweet taste liking. Drinks were rated as more intensely sweet with increasing sugar concentration. The drinks with the least and the most amount of added sugar were liked less $(p<0.05)$. did not show differences between the drinks, $\chi^{2}(6)=11.10, p=0.085, \chi^{2}(6)=12.73, p=0.048$, and $\chi^{2}(6)=12.51, p=0.051$ for THC, CBD, and placebo, respectively.

\section{Appetite and ad libitum intake}

Results from the appetite questionnaire can be found in Figure 4. Reported hunger did not differ between conditions, $F(2,18)=2.62, p=0.10$, and did not change over time, $F(2,18)=2.47, p=0.11$. However, reported fullness differed between conditions, $F(2,18)=3.74$, $p<0.05$, and changed over time, $F(2,18)=4.38, p<$ 0.05 . Throughout the whole test session, fullness ratings were higher in the CBD condition than in the THC condition. Desire to eat differed between conditions, $F(2,18)=6.50, p<0.05$, and was higher in the THC condition than in the CBD condition throughout the whole test session, but did not change over time, $F(2,18)=2.43, p=0.12$. Prospective consumption differed between conditions, $F(2,18)=4.83, p<0.05$, but did not change over time, $F(2,18)=3.34, p=0.06$.

Ad libitum intake of the most liked chocolate milklike drink did not differ between conditions, $F(2,18)=$ $0.50, p=0.62$. Participants consumed on average $537 \pm$ $67 \mathrm{kcal}$ in the THC condition, $581 \pm 66 \mathrm{kcal}$ in the CBD condition, and $595 \pm 65 \mathrm{kcal}$ in the placebo condition.

\section{Food preferences}

The preference scores can be found in Table 3. Treatments did not affect preference for sweet or for any of the macronutrients, all $p>0.05$. In all three conditions, participants had a preference for savory over sweet, all $p<0.05$, but not for one of the macronutrients over the others, all $p>0.05$.

\section{Discussion}

To the best of our knowledge, this study is the first randomized, placebo-controlled double-blind study investigating sweet taste intensity perception, sweet taste liking, and possible consequent changes in food preference following inhalation of THC and CBD. The results indicate no change in sweet taste intensity perception and liking, nor in food preferences, as related to taste (sweet vs. savory) and macronutrient composition (carbohydrate, protein, fat, and low energy).

The lack of effects on sweet taste intensity and liking after activating or inhibiting the ECS is contradictory to our expectations based on previous studies that showed endocannabinoid modulation of sweet taste intensity perception in mice. ${ }^{19,20}$ However, the current findings 

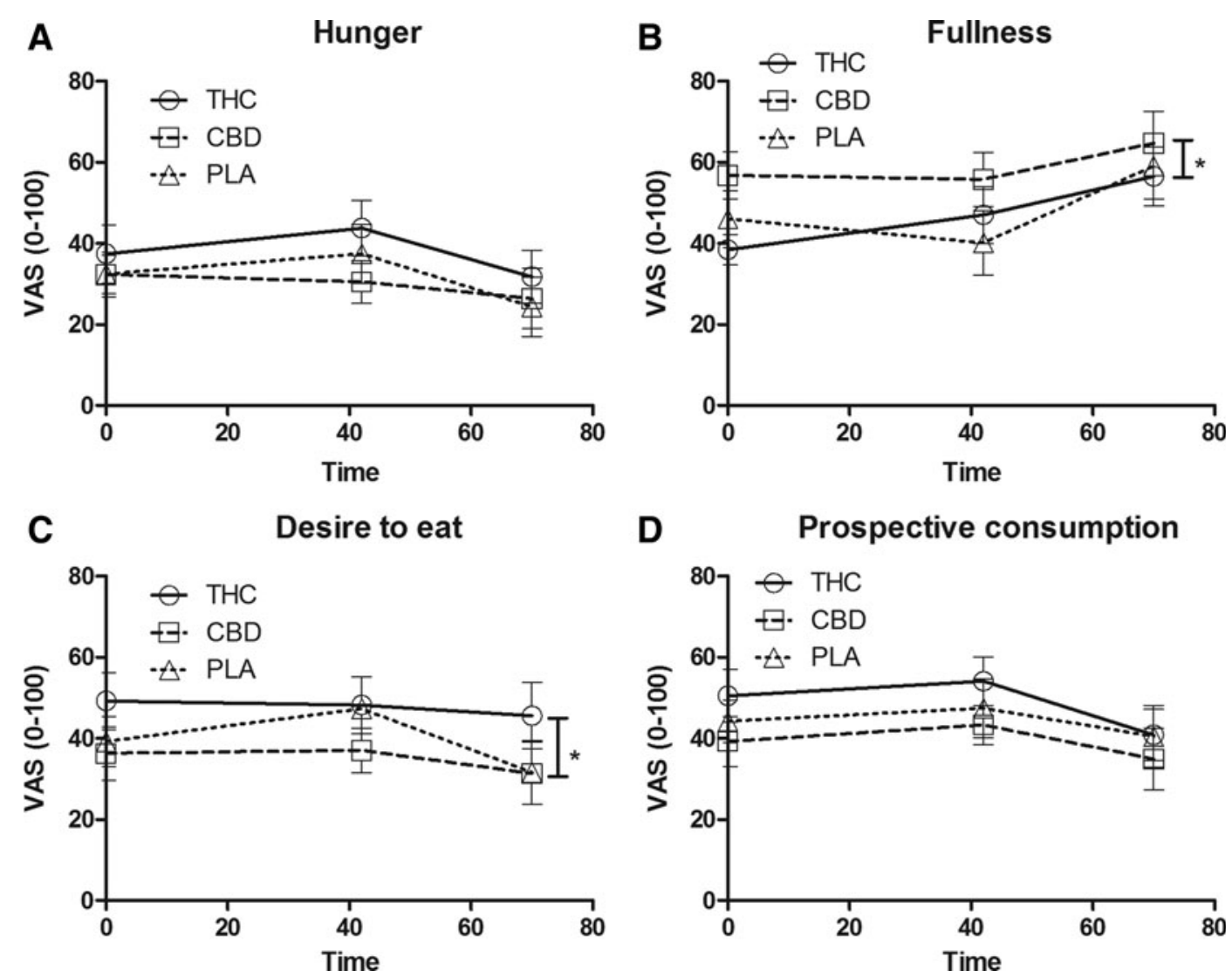

FIG. 4. Rated scores (mean \pm SEM) for (A) hunger, (B) fullness, (C) desire to eat, and (D) prospective consumption. *Indicates difference throughout THC and CBD conditions $(p<0.05)$.

are in line with a study in humans that assessed taste intensity perception after oral THC administration. In that study, THC did not affect intensity and liking of sweetness, saltiness, sourness, and bitterness. ${ }^{28}$

In line with the results from the taste test, preference for sweet taste in the MTPRT did not differ between the THC, CBD, and placebo conditions. In addition, preference for macronutrients was not affected by the administration of THC or CBD. Earlier

Table 3. Food Preference Scores in All Three Conditions

\begin{tabular}{lccc}
\hline & THC & CBD & Placebo \\
\hline High carbohydrate & $2.39 \pm 0.07$ & $2.30 \pm 0.13$ & $2.43 \pm 0.14$ \\
High fat & $2.61 \pm 0.15$ & $2.42 \pm 0.12$ & $2.42 \pm 0.11$ \\
High protein & $2.74 \pm 0.26$ & $2.74 \pm 0.20$ & $2.84 \pm 0.19$ \\
Low energy & $2.26 \pm 0.25$ & $2.54 \pm 0.26$ & $2.31 \pm 0.24$ \\
Sweet & $2.20 \pm 0.09$ & $2.22 \pm 0.08$ & $2.20 \pm 0.10$
\end{tabular}

Values are mean \pm SEM. Preference scores for macronutrient categories can range between 1 and 4, preference score for sweet can range between 1.5 and 3.5 . studies in humans showed that Cannabis increases intake of snack foods, with a specific increased intake of sweet snacks. ${ }^{21,22}$

In this study, participants had a preference for savory over sweet. Just before completing the MTPRT, participants had consumed a large amount of a sweet drink to satiety. This might have caused sensory-specific satiety, that is, decreased pleasantness of sensory properties of the food that has just been consumed to satiety. ${ }^{36}$ The effect of sensory-specific satiety might have overruled a potential effect of endocannabinoid modulation on preference for sweet taste. However, given that THC and CBD administration did not influence sweet taste intensity perception and liking of the chocolate milklike drinks, we think it is more likely that there is no effect of endocannabinoid modulation on sweet taste preference.

Desire to eat was higher and fullness was lower in the THC condition compared to the CBD condition, possibly 
related to baseline differences. However, despite these differences, ad libitum intake of the most preferred chocolate milk-like drink did not differ between conditions. In a previous study in cancer patients, THC treatment increased appetite, but did not affect total energy intake. ${ }^{37}$ Also, in a healthy population, THC did not affect the total energy intake. ${ }^{38}$ However, other studies in humans showed that THC increases food intake. ${ }^{21,22}$ CBD has previously been shown not to affect appetite in patients who suffer from type 2 diabetes. ${ }^{39}$ In rats, however, CBD has been shown to decrease food intake, ${ }^{12,13}$ which may be caused by higher fullness. These contradicting findings warrant further investigation.

The plasma levels of THC and CBD showed that the administration of pharmacological doses by inhalation was successful in our study. The placebo Cannabis contained traces of THC, which led to detectable, but very low, THC plasma levels in the placebo condition. As THC plasma levels in the placebo condition were eight times lower than in the THC condition, the functional consequences on outcome measures are likely minimal.

The relatively small sample size could be regarded as a limitation of our exploratory study. However, we would expect to find similar results in a larger sample. With 10 participants, we see a great overlap of the mean sweet taste intensity and liking of the different drinks, which suggests that we will also not see any differences with more participants included in the study. Especially for the sweet taste liking, there is a large variation between participants. For the food preference task, one may expect to find differences in preference for low-energy products with a larger sample size. As CBD increases fullness and decreases desire to eat, we would expect that it also increases preference for low-energy products based on previous experience with the MTPRT. ${ }^{30}$

This study included only male participants. As endocannabinoid pharmacology differs between males and females, ${ }^{40,41}$ females might respond differently to inhaled THC and CBD. Future studies should take this into account and consider to include both males and females.

Another plausible explanation for our negative results might be that we used a relatively low dose of THC compared to other studies. ${ }^{31,32}$ We chose this dose as we did not want obvious intoxication to influence the results. However, it is possible that the dose is too low to evoke an effect and we would have seen an effect of THC on sweet taste intensity perception and liking if we would have used a higher dose. Importantly, the effects of THC and other $\mathrm{CB}_{1}$ agonists usually follow an inverted $\mathrm{u}$-shape, that is, there is an optimal dose at which the largest effect occurs and for higher doses, the effect wears off. ${ }^{5,17,42}$ Dosing too high could therefore also lead to no effect. Future studies should take this in consideration.

Based on the results from this study, we conclude that challenging the ECS with low doses of THC or CBD does not influence sweet taste intensity perception and liking in humans. In addition, low doses of THC and CBD do not affect food preferences.

\section{Acknowledgments}

We would like to thank Pauline Claessen, research dietician, for providing the recipe for the chocolate milklike drinks, and Tim Crolla and Michele Pedrotti for their help in carrying out the study. Research presented in this publication was financially supported by the Graduate School VLAG.

\section{Author Disclosure Statement}

No competing financial interests exist.

\section{References}

1. Lowe MR, Butryn ML. Hedonic hunger: a new dimension of appetite? Physiol Behav. 2007;91:432-439.

2. Droste SM, Saland SK, Schlitter EK, et al. AM 251 differentially effects foodmaintained responding depending on food palatability. Pharmacol Biochem Behav. 2010;95:443-448.

3. DiPatrizio NV, Astarita G, Schwartz G, et al. Endocannabinoid signal in the gut controls dietary fat intake. Proc Natl Acad Sci U S A. 2011; 108:12904-12908.

4. Argueta DA, DiPatrizio NV. Peripheral endocannabinoid signaling controls hyperphagia in western diet-induced obesity. Physiol Behav. 2017;171:32-39.

5. DiPatrizio NV, Simansky KJ. Activating parabrachial cannabinoid CB1 receptors selectively stimulates feeding of palatable foods in rats. J Neurosci. 2008;28:9702-9709.

6. Cooper SJ. Endocannabinoids and food consumption: comparisons with benzodiazepine and opioid palatability-dependent appetite. Eur J Pharmacol. 2004;500:37-49.

7. Rogers PJ. Why a palatability construct is needed. Appetite. 1990;14:167170.

8. de Graaf C, van Staveren W, Burema J. Psychophysical and psychohedonic functions of four common food flavours in elderly subjects. Chem Senses. 1996;21:293-302.

9. O'Brien LD, Wills KL, Segsworth B, et al. Effect of chronic exposure to rimonabant and phytocannabinoids on anxiety-like behavior and saccharin palatability. Pharmacol Biochem Behav. 2013;103:597-602.

10. De Luca MA, Solinas M, Bimpisidis Z, et al. Cannabinoid facilitation of behavioral and biochemical hedonic taste responses. Neuropharmacology. 2012;63:161-168.

11. Higgs S, Williams CM, Kirkham TC. Cannabinoid influences on palatability: microstructural analysis of sucrose drinking after $\Delta 9$ tetrahydrocannabinol, anandamide, 2-arachidonoyl glycerol and SR141716. Psychopharmacology (Berl). 2003;165:370-377.

12. Farrimond JA, Whalley BJ, Williams CM. Cannabinol and cannabidiol exert opposing effects on rat feeding patterns. Psychopharmacology (Berl). 2012;223:117-129.

13. Wierucka-Rybak M, Wolak M, Bojanowska $E$. The effects of leptin in combination with a cannabinoid receptor 1 antagonist, AM 251, or cannabidiol on food intake and body weight in rats fed a high-fat or a freechoice high sugar diet. J Physiol Pharmacol. 2014;65:487-496. 
14. Pertwee RG. The diverse $\mathrm{CB} 1$ and $\mathrm{CB} 2$ receptor pharmacology of three plant cannabinoids: $\Delta(9)$-tetrahydrocannabinol, cannabidiol and $\Delta 9$ tetrahydrocannabivarin. Br J Pharmacol. 2008;153:199-215.

15. McPartland JM, Duncan M, Di Marzo V, et al. Are cannabidiol and Delta9tetrahydrocannabivarin negative modulators of the endocannabinoid system? A systematic review. Br J Pharmacol. 2015;172:737-753.

16. Riedel G, Fadda P, McKillop-Smith $S$, et al. Synthetic and plant-derived cannabinoid receptor antagonists show hypophagic properties in fasted and non-fasted mice. Br J Pharmacol. 2009;156:1154-1166.

17. Wiley JL, Burston JJ, Leggett DC, et al. CB 1 cannabinoid receptormediated modulation of food intake in mice. $\mathrm{Br} \mathrm{J}$ Pharmacol. 2005; 145:293-300.

18. Ligresti A, De Petrocellis L, Di Marzo V. From phytocannabinoids to cannabinoid receptors and endocannabinoids: pleiotropic physiological and pathological roles through complex pharmacology. Physiol Rev. 2016;96:1593-1659.

19. Yoshida R, Ohkuri T, Jyotaki $M$, et al. Endocannabinoids selectively enhance sweet taste. Proc Natl Acad Sci U S A. 2010;107:935-939.

20. Niki $M$, Jyotaki $M$, Yoshida $R$, et al. Modulation of sweet taste sensitivities by endogenous leptin and endocannabinoids in mice. J Physiol. 2015;593:2527-2545.

21. Abel EL. Cannabis: effects on hunger and thirst. Behav Biol. 1975;15:255-281.

22. Foltin RW, Fischman MW, Byrne MF. Effects of smoked marijuana on food intake and body weight of humans living in a residential laboratory. Appetite. 1988;11:1-14.

23. Tart CT. Marijuana intoxication: common experiences. Nature. 1970;226:701-704.

24. Hart CL, Ward AS, Haney M, et al. Comparison of smoked marijuana and oral $\Delta$ 9-tetrahydrocannabinol in humans. Psychopharmacology (Berl). 2002; 164:407-415

25. Smit E, Crespo CJ. Dietary intake and nutritional status of US adult marijuana users: results from the Third National Health and Nutrition Examination Survey. Public Health Nutr. 2001;4:781-786.

26. Pi-Sunyer FX, Aronne LJ, Heshmati HM, et al. Effect of rimonabant, a cannabinoid-1 receptor blocker, on weight and cardiometabolic risk factors in overweight or obese patients: RIO-North America: a randomized controlled trial. JAMA. 2006;295:761-775.

27. Van Gaal LF, Rissanen AM, Scheen AJ, et al. Effects of the cannabinoid-1 receptor blocker rimonabant on weight reduction and cardiovascular risk factors in overweight patients: 1-year experience from the RIO-Europe study. Lancet. 2005;365:1389-1397.

28. Mattes RD, Shaw LM, Engelman K. Effects of cannabinoids (marijuana) on taste intensity and hedonic ratings and salivary flow of adults. Chem Senses. 1994;19:125-140.

29. Van Strien T, Frijters JER, Bergers GPA, et al. The Dutch eating behavior questionnaire (DEBQ) for assessment of restrained, emotional, and external eating behavior. Int J Eat Disord. 1986;5:295-315.

30. de Bruijn SEM, de Vries YC, de Graaf $C$, et al. The reliability and validity of the Macronutrient and Taste Preference Ranking Task: a new method to measure food preferences. Food Qual Prefer. 2017;57:32-40.

31. Kowal MA, Hazekamp A, Colzato LS, et al. Cannabis and creativity: highly potent Cannabis impairs divergent thinking in regular Cannabis users. Psychopharmacology (Berl). 2015;232:1123-1134.

32. Kleinloog D, Roozen F, De Winter W, et al. Profiling the subjective effects of $\Delta$ 9-tetrahydrocannabinol using visual analogue scales. Int J Methods Psychiatr Res. 2014;23:245-256.
33. Schutz HG, Cardello AV. A labeled affective magnitude (LAM) scale for assessing food liking/disliking. J Sens Stud. 2001;16:117-159.

34. Green BG, Shaffer GS, Gilmore MM. Derivation and evaluation of a semantic scale of oral sensation magnitude with apparent ratio properties. Chem Senses. 1993;18:683-702.

35. Blundell J, De Graaf C, Hulshof T, et al. Appetite control: methodological aspects of the evaluation of foods. Obes Rev. 2010;11:251-270.

36. Rolls $B J$, Rolls ET, Rowe EA, et al. Sensory specific satiety in man. Physiol Behav. 1981;27:137-142.

37. Brisbois TD, de Kock IH, Watanabe SM, et al. Delta-9tetrahydrocannabinol may palliate altered chemosensory perception in cancer patients: results of a randomized, double-blind, placebocontrolled pilot trial. Ann Oncol. 2011;22:2086-2093.

38. Mattes RD, Engelman K, Shaw LM, et al. Cannabinoids and appetite stimulation. Pharmacol Biochem Behav. 1994:49:187-195.

39. Jadoon KA, Ratcliffe SH, Barrett DA, et al. Efficacy and safety of cannabidiol and tetrahydrocannabivarin on glycemic and lipid parameters in patients with type 2 diabetes: a randomized, double-blind, placebocontrolled, parallel group pilot study. Diabetes Care. 2016;39:17771786.

40. Craft RM, Marusich JA, Wiley JL. Sex differences in cannabinoid pharmacology: a reflection of differences in the endocannabinoid system? Life Sci. 2013;92:476-481.

41. Diaz S, Farhang B, Hoien J, et al. Sex differences in the cannabinoid modulation of appetite, body temperature and neurotransmission at POMC synapses. Neuroendocrinology. 2009;89:424-440.

42. Bellocchio $L$, Lafentre $P$, Cannich $A$, et al. Bimodal control of stimulated food intake by the endocannabinoid system. Nat Neurosci. 2010;13:281-283.

Cite this article as: de Bruijn SEM, de Graaf C, Witkamp RF, Jager G (2017) Explorative placebo-controlled double-blind intervention study with low doses of inhaled $\Delta 9$-tetrahydrocannabinol and cannabidiol reveals no effect on sweet taste intensity perception and liking in humans, Cannabis and Cannabinoid Research 2:1, 114-122, DOI: 10.1089 /can.2017.0018.

$\begin{aligned} & \text { Abbreviations Used } \\ & \text { ANOVA }=\text { analysis of variance } \\ & \mathrm{CBD}=\text { cannabidiol } \\ & \mathrm{ECS}=\text { endocannabinoid system } \\ & \mathrm{MTPRT}=\text { Macronutrient and Taste Preference Ranking Task } \\ & \mathrm{ND}=\text { not detected } \\ & \mathrm{OMC}=\text { Office for Medicinal Cannabis } \\ & \mathrm{SEM}=\text { standard error of the mean } \\ & \mathrm{THC}=\Delta \text { 9-tetrahydrocannabinol } \\ & \mathrm{VAS}=\text { Visual Analogue Scales }\end{aligned}$

\section{Publish in Cannabis and Cannabinoid Research}

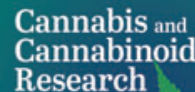

- Immediate, unrestricted online access

- Rigorous peer review

- Compliance with open access mandates

- Authors retain copyright

- Highly indexed

- Targeted email marketing 